\title{
EchoGéo
}

$28 \mid 2014$

Police : les espaces de l'ordre, l'ordre en espace

\section{La délinquance : observatoire méconnu des recompositions des relations villes-campagnes}

Jorick Perrin

\section{OpenEdition}

1 Journals

Édition électronique

URL : https://journals.openedition.org/echogeo/13812

DOI : 10.4000/echogeo.13812

ISSN : 1963-1197

Éditeur

Pôle de recherche pour l'organisation et la diffusion de l'information géographique (CNRS UMR 8586)

Référence électronique

Jorick Perrin, «La délinquance : observatoire méconnu des recompositions des relations villescampagnes », EchoGéo [En ligne], 28 | 2014, mis en ligne le 08 juillet 2014, consulté le 31 juillet 2021. URL : http://journals.openedition.org/echogeo/13812 ; DOI : https://doi.org/10.4000/echogeo.13812

Ce document a été généré automatiquement le 31 juillet 2021.

EchoGéo est mis à disposition selon les termes de la licence Creative Commons Attribution - Pas d'Utilisation Commerciale - Pas de Modification 4.0 International (CC BY-NC-ND) 


\title{
La délinquance : observatoire méconnu des recompositions des relations villes-campagnes
}

\author{
Jorick Perrin
}

\section{Introduction}

1 La délinquance s'invite dans les campagnes. Derrière ce titre aguicheur, relayé par de nombreux médias régionaux et nationaux, se cache une réalité complexe mêlant des données géographiques diverses. En effet, depuis plusieurs années, certains journalistes font écho d'un déplacement de la délinquance de la ville vers la campagne. Cet imaginaire d'une délinquance exclusivement urbaine par le passé et qui s'étendrait aux espaces périurbains et ruraux, se répand auprès des populations et présente de multiples conséquences, parfois ubuesques, comme le développement de caméras de surveillance dans des communes où aucun acte de délinquance n'est constaté. Or, aucun travail scientifique n'a à ce jour révélé l'existence d'un tel déplacement de la délinquance. La géographie ne semble pas avoir pris conscience de la portée des thématiques liées à la sécurité et aux phénomènes délictuels dans lesquels la composante spatiale tient une part importante. En effet, ces objets sont analysés par des juristes, des sociologues, des psychiatres, mais très peu par des géographes à l'exception de Gérard Camilleri et de Christine Lazerges qui, en 1992, ouvrent le champ d'une "géographie criminelle» (Camilleri et Lazerges, 1992) ainsi que de Bernard Rémy sur le territoire de la Réunion (Rémy, 2000). Ce paradoxe, entre un processus d'actualité largement médiatisé et une absence de recherches en géographie " criminelle », pose la question de la pertinence scientifique de cet imaginaire.

2 La recherche d'une définition géographique de la délinquance apparaît comme une première difficulté. Parmi les dictionnaires courants de géographie en langue française, seul le dictionnaire d'Yves Lacoste propose une entrée du terme délinquance ${ }^{1}$. Il la définit comme : 
«l'ensemble des crimes et délits [...]. D'un point de vue géographique, la délinquance peut être envisagée à différents niveaux d'analyse spatiale, en faisant la différence entre villes et campagnes [...]. La délinquance est aussi à examiner au plan international notamment pour les trafics de drogues, avec leurs diverses routes clandestines qui se raccordent aux multiples réseaux de distribution locale, sans oublier les paradis fiscaux où se recycle l'argent sale » (Lacoste, 2003).

Cette approche multiscalaire montre les possibilités dont dispose la géographie pour aborder ces thématiques que ce soit sur la répartition spatiale du crime, sur les effets de lieux et de concentration mais aussi sur la dimension socio-spatiale de la pratique criminelle elle-même. Malgré cette timidité des géographes relevée par Roger Brunet, certaines études effleurent les "déviances négatives» au travers de travaux sur les exclusions, les marginalités, ainsi que les pauvretés (Brunet, 1989). D'autres tels Patrick Pigeon ou encore Julien Rebotier s'en approchent davantage au sein de la géographie des risques, en travaillant sur certaines caractéristiques particulières de la délinquance (Pigeon, 2005; Rebotier, 2008). Pour autant, cette situation française ne caractérise pas les géographes anglo-saxons, comme Sampson et Groves en 1989 (théorie de la désorganisation sociale), Sherman en 1995 (hotspots) ou encore Newman en 1996 (espace défendable), qui produisent des résultats liant la délinquance et la composante spatiale. Ils ont ainsi établi des modèles théoriques corrélant la distance aux domiciles et l'intensité des «incidents» (Besson, 2004). Malgré la quasi-absence de géographes travaillant sur ces questions en France, la localisation de la délinquance reste un outil utilisé par les acteurs de la sécurité ainsi que par des organismes de recherche comme l'observatoire national de la délinquance et des réponses pénales (ONDRP) qui publie chaque année une " géographie criminelle de la France $»^{2}$.

Pourtant la géographie dispose d'atouts considérables pour traiter de ces problématiques. Les outils comme la carte, les SIG, mais aussi ses paradigmes comme l'analyse spatiale peuvent fournir des clefs de compréhension voire des modélisations de phénomènes s'inscrivant dans un espace qui par définition n'est pas neutre. La cartographie statistique est ainsi utilisée par divers acteurs afin de construire ou de diffuser un savoir sur un fait social spatialisé. De ce fait, une réflexion préalable est nécessaire à tout travail de cartographie statistique (objectifs, public, collecte du contenant, choix de représentations graphiques, etc.). La cartographie thématique apparait ainsi non pas comme un cumul de l'information mais comme une sélection de celle-ci, un classement et un traitement qui la transforme, toute représentation constituant une interprétation du réel (Monmonier, 1991) ${ }^{3}$.

De cette manière, la représentation de la délinquance s'insère dans le cadre de la réflexion sur les fonctions des cartes. En effet, la carte en tant que source de données et outil de repérage permet de localiser la distribution spatiale d'un phénomène (vols, cambriolages, etc.). Ensuite, la carte peut s'utiliser comme un outil d'exploration permettant la révélation de relations spatiales. Puis elle s'intègre parmi différents véhicules de communication afin de transmettre un message. L'accent peut ainsi être mis sur la sémiotique afin de s'adapter au public concerné (politiques, usages éducatifs, etc.). Enfin, elle peut s'employer comme un outil de concertation. Construction opérationnelle pour des professionnels, elle sert alors à conduire des échanges et à prendre des décisions (par exemple en contrecarrant les facteurs favorisant les phénomènes délictuels).

6 L'espace n'apparait pas uniquement comme un simple cadre mais il agit également sur les phénomènes délictuels que ce soit en matière d'aménagement (espace défendable) 
mais aussi en ce qui concerne la perception à travers l'espace vécu, ou encore la théorie de la vitre brisée théorisée par George L. Kelling (Kelling, 1996) et développée en France par Sébastian Roché (Roché, 2002). De plus, l'espace est le théâtre de multiples interactions sous l'effet de la métropolisation. Ces transformations affectent aussi bien les territoires urbains que ruraux au travers des évolutions du prix du foncier, du développement de l'offre de transports, ou encore par la diffusion d'un imaginaire mettant en avant un mode de vie plus proche de la nature au sein des campagnes.

7 Le développement des modes de vie urbains dans des espaces périurbains et ruraux pourrait ainsi affecter la géographie de la délinquance en raison de l'avènement de l'individualisme, source de diminution de l'interconnaissance dans les villages que cela signifie, voire également par l'explosion des mobilités. En effet, des délinquants se déplacent-ils parmi ces néo-ruraux ? Ou bien s'agit-il d'une imitation des modes de vie urbains, et de fait de sa délinquance, qui exercerait une forte attractivité auprès des populations périurbaines? Ou encore existe-t-il un gradient de distance par rapport à la ville, certains types de délinquance se diffusant en fonction du poids de la ville? Ces quelques hypothèses demandent à être vérifiées par des études intégrant les facteurs spatiaux et territoriaux qui évoluent avec la complexification des relations villescampagnes. Il s'agit donc au travers de cette étude, de voir en quoi l'essor d'une "nouvelle délinquance », autrement dit d'actes délictuels potentiellement marqués par le fait urbain et émergeant dans ces espaces périurbains et ruraux, servirait d'observatoire des transformations des relations villes-campagnes.

8 Pour ce faire, il est nécessaire d'aborder les possibilités et les réalités offertes par le lien géographie-délinquance dans le débat scientifique. Puis le développement doit s'enrichir de l'étude de l'influence de l'urbain pour différents types de délinquance afin de déterminer l'existence ou non d'une délinquance spécifiquement urbaine, périurbaine ou rurale. Enfin, il convient de caractériser l'influence de l'urbain sur la délinquance présente dans les espaces périurbains et ruraux.

\section{La relation géographie-délinquance : quelles réalités, quelles possibilités?}

\section{Les recompositions des relations villes-campagnes font émerger de nouvelles formes de délinquance dans des espaces ruraux}

Les mutations socio-fonctionnelles à l'œuvre dans les relations villes-campagnes engendrent des modifications sur de nombreux plans, qu'ils soient matériels ou conceptuels. Ainsi, l'essor d'une population marquée par des modes de vie urbains au sein des espaces périurbains et des campagnes affecte les pratiques rurales présentes dans ces territoires. Cependant, toutes les campagnes et toutes les villes ne subissent pas ce processus de manière uniforme et identique. La délinquance et ses évolutions peuvent alors apparaître comme une illustration de ces transformations à plusieurs égards.

10 La transition d'une société rurale à une société urbaine moderne s'est traduite au début $\mathrm{du} \mathrm{XX}^{\mathrm{e}}$ siècle par la modification de certains rapports sociaux. Ainsi, les sociétés rurales étaient basées sur l'interconnaissance, créant ainsi une contrainte de proximité pour les délinquants (Robert, 2003). Or, l'hypermobilité qui caractérise la société urbaine 
différencie potentiellement les espaces de commission d'actes délictueux et les espaces d'origine des délinquants. L'intensification des flux s'observe à l'échelle de vie des individus. En effet, l'augmentation des flux domicile-travail montre une concentration des emplois dans les pôles urbains, associée à un déplacement progressif de la population vers les zones périurbaines. Ce processus nécessite une réponse adaptée des forces de l'ordre au sein de ces espaces, mais aussi le long des voies de circulation (Garcin, 2005).

11 La prise en compte géographique de la délinquance se trouve confrontée aux transformations socio-spatiales des espaces ruraux et périurbains. En effet, des problématiques délictuelles nommément liées à la ville comme les «violences urbaines » se retrouvent en fait également en dehors des espaces qui les définissent (Bui Trong, 1998). Les transformations que nous venons d'évoquer concernent les espaces ruraux et périurbains qui présentent un lien étroit avec la ville. Inversement, certains espaces ruraux sont marqués par une régression démographique et un vieillissement de la population (Dieu, 2002). Cette précarisation des populations s'observe dans le rural profond, autrement dit, dans des zones rurales enclavées, où les services publics se raréfient et où les activités économiques des principaux centres urbains sont éloignées et difficilement accessibles. La brigade de gendarmerie tient ainsi un rôle social qui dépasse sa simple mission de sécurité et elle se positionne alors comme l'unique instrument de l'État (Mouhanna, 2001 ; Dieu, 2009). Au sein de ces espaces ruraux en décroissance, les forces de l'ordre, en l'occurrence la Gendarmerie nationale, doivent également s'adapter à l'essor d'une population plus vulnérable et donc à une délinquance spécifique. L'affirmation de ces nouveaux territoires nécessite une analyse fine des processus en cours afin d'en cerner les composantes et les enjeux ${ }^{4}$. La demande et la production de sécurité ne sont donc pas homogènes dans les territoires ruraux.

12 Les recompositions des relations villes-campagnes transforment les territoires et les pratiques délictuelles qui y sont associées. Si ce processus est pris en compte par les acteurs de la sécurité, les études géographiques semblent absentes de cette thématique. Or les outils du géographe, et notamment la cartographie, sont largement utilisés dans le cadre de la diffusion d'un message politico-médiatique.

\section{Une cartographie de la délinquance largement développée au service d'un message politico-médiatique}

La délinquance est un sujet particulièrement porteur depuis deux décennies dans le débat public français. Que ce soit par les hommes politiques ou par les médias, les thématiques liées aux phénomènes délictuels sont soumises à diverses tentatives d'appropriation au nom d'une prétendue vérité scientifique. Nous ne reviendrons pas ici sur l'utilisation des statistiques de la délinquance et sur leurs limites, qui ont par ailleurs largement été analysées (Robert et al. 1994 ; Mucchielli, 2002 ; Mattelly, 2007). Cependant, une nouvelle donnée tend à se diffuser dans les messages politiques et médiatiques, à savoir celle de l'espace et notamment de la cartographie de la délinquance.

En effet, le paramètre géographique peut être instrumentalisé politiquement afin d'obtenir des modifications sur la perception voire la compréhension de la délinquance. Ces transformations visibles en géographie électorale (gerrymandering) visent à 
modifier les mailles géographiques afin de favoriser un parti. Dans le domaine des déviances négatives, cette délimitation géographique s'observe dans un premier temps au travers de la communication des chiffres de la délinquance. Ces derniers sont diffusés par le ministère de l'Intérieur et ils ne sont pas accessibles à toutes les échelles géographiques. Ainsi, l'arrondissement correspond à l'échelon territorial le plus fin auquel les données sont transmises. Ces arrondissements correspondent généralement aux découpages entre les compagnies de gendarmerie nationale - pour les zones rurales et périurbaines - et les circonscriptions de sécurité publique - à savoir les zones urbaines - sous compétence de la police nationale. Le fait de ne pas pouvoir disposer de données spatiales plus précises, telles que les communes voire les quartiers, limite la pertinence des résultats puisque la réalité géographique de ces phénomènes peut varier à une échelle micro. Ces données existent, mais ne sont pas diffusées afin d'éviter notamment de potentielles concurrences entre communes. Leur diffusion pose en effet des problèmes politiques et éthiques (Ghorra-Gobin, 2011). Comme le rappelle Armand Frémont, elle pourrait "amplifier des phénomènes de ségrégation sociale », ainsi que « servir des idéologies extrêmes » (Frémont, 2005).

«La cartographie, aveuglante parfois de trop de vérité, manipulable et éventuellement manipulée, apparait bien comme un enjeu entre autorité et liberté. Un enjeu militaire, technique, politique, mais aussi social. Ainsi, comme pour les autres représentations, s'impose peu à peu l'idée d'une réflexion éthique sur la cartographie, et, par voie de conséquence, sur la géographie » (Frémont, 2005, p. 42-43).

Depuis 2003, l'Observatoire national de la délinquance et des réponses pénales (ONDRP) est responsable de l'étude des phénomènes délictuels en tant que département de l'Institut national des hautes études de la sécurité et de la justice (INHESJ). Ce dernier est un établissement public chargé de promouvoir et de diffuser les informations notamment en matière de sécurité intérieure et de justice. Pourtant son intérêt pour la géographie de la délinquance est très récent. En effet, l'ONDRP qui publie des rapports annuels sur l'état de la délinquance en France propose une cartographie " criminelle » uniquement depuis 2007 au sein du dit rapport (Perrin, 2013). S'ajoute à cela la création en 2009, par ce même organisme, d'un site internet (www.cartocrime.net) permettant de réaliser ses propres cartes sur la délinquance à l'échelle régionale ou départementale. Pour autant, cette prise en compte spatiale de la délinquance reste d'un intérêt limité puisque les cartes ne sont pas accompagnées d'études ou de commentaires qui permettraient d'éclairer le citoyen sur ces données géographiques.

La cartographie de la délinquance est susceptible de subir diverses instrumentalisations politiques. C'est un outil d'autant plus redoutable que son impact se trouve plus ou moins amplifié selon le récepteur. En effet, contrairement à d'autres types de vecteurs, les connaissances et les compétences permettant d'effectuer une analyse critique des cartes sont peu répandues auprès des citoyens (Monmonier, 1991).

Les médias peuvent également être sujets à ces tentatives de déformation de données géographiques. En effet, la compétition médiatique de l'exclusivité peut entraîner les journalistes à rechercher des caractéristiques spectaculaires d'un événement, ce qui leur permet d'attirer les lecteurs, auditeurs ou téléspectateurs (Champagne, 1993). Cette « dictature de l'audimat » s'avère particulièrement visible au travers des médias télévisuels qui associent des successions d'images au discours journalistique (Noseda, 2005). Ainsi, ce dynamisme audiovisuel, caractérisant la temporalité médiatique contemporaine, complexifie la prise de recul nécessaire à une analyse critique de 
l'information. L'accent serait davantage mis sur les évènements les plus graves et les plus spectaculaires plutôt que sur les faits de masse, plus réguliers (Winick, 1978). La sélection de l'information la plus grave puis sa diffusion entraîne un effet anxiogène voire une culture de la peur auprès de la population, ce qui participe à la production du sentiment d'insécurité (Moisi, 2008). D'un point de vue formel, les moyens de représentation d'un phénomène au sein des thématiques liées à la délinquance peuvent accentuer ce sentiment anxiogène, et notamment via l'utilisation de cartes (Giblin (dir.), 2009).

Les études sur la délinquance et plus précisément sur sa répartition géographique sont affectées par des tentatives d'appropriation et d'instrumentalisation qui nuisent à une recherche scientifique rigoureuse. Pour autant, la géographie se doit de participer à l'établissement de connaissances scientifiques dans le domaine de la délinquance. Une première difficulté apparaît dans cet objectif, à savoir la détermination des espaces « urbains », « périurbains » et « ruraux ».

\section{L'utilisation des zones polices et gendarmerie pour qualifier l'urbain}

19 Il convient avant tout de préciser la méthodologie de l'étude qui a conduit à une analyse des recompositions des relations villes-campagnes au prisme de la délinquance. En effet, l'objectif de l'étude est multiple. D'une part, elle se doit de déterminer l'existence ou non d'une délinquance urbaine et d'une délinquance périurbaine, voire rurale, et de caractériser les infractions (cf. Quelle place de l'urbanité dans l'étude de la délinquance?). D'autre part, au sein des espaces ruraux et périurbains, elle doit analyser l'influence de l'urbain sur chaque type de délinquance (cf. La délinquance périurbaine et rurale sous influence urbaine). Il convient à présent de revenir sur les choix méthodologiques qui ont été faits afin d'atteindre ces objectifs.

L'étude se situe dans le cadre théorique de l'analyse spatiale visant à la recherche de lois, de règles générales de fonctionnement de l'espace afin de comprendre celui-ci. Ainsi, la délinquance, abordée comme interaction spatiale, comporte des constantes qu'il convient de définir ainsi que des caractéristiques résiduelles dont l'analyse et l'explication sont nécessaires.

21 Le cadre spatial est celui des départements de la France métropolitaine. Une étude plus fine pourrait être réalisée et se rapprocher davantage de la réalité géographique, mais elle perdrait en lisibilité car elle ne permettrait pas de dégager de grandes tendances à l'échelle régionale et nationale. Temporellement, l'étude utilise des données du ministère de l'Intérieur datant de 2009. En effet, 2009 est la dernière année où le découpage des statistiques se fait sous une présentation "classique» facilitant le découpage des infractions pénales en catégories plus vastes afin d'améliorer la lecture du phénomène. Les statistiques de la délinquance sont présentées au sein d'un fichier nommé « état 4001 » qui regroupe 107 index correspondant aux infractions pénales. Il est ainsi nécessaire de réaliser des regroupements afin d'établir des catégories de délinquance. L'étude reprend les regroupements établis par le ministère de l'Intérieur dans l'état 4001 et en recense neuf :

- la délinquance totale

- la délinquance de voie publique

- les infractions révélées par l'action des services

- les atteintes volontaires à l'intégrité physique 
- les atteintes aux biens

- les vols

- les escroqueries et infractions économiques et financières

- les crimes et délits contre les personnes

- les autres infractions

Enfin, il reste à déterminer les traits urbains, périurbains ou ruraux de chaque département. Pour ce faire, l'étude se base sur les caractéristiques des statistiques du ministère de l'Intérieur, à savoir la différenciation entre la délinquance constatée par la police et la gendarmerie. En effet, la police est responsable de l'ordre public des espaces urbains de plus de 20000 habitants, tandis que la Gendarmerie nationale sécurise le reste du territoire national. L'étude assimile ainsi la délinquance des villes à la délinquance constatée par les services de la Police nationale, et la délinquance des espaces périurbains et ruraux à celle constatée par les unités de la Gendarmerie nationale ${ }^{5}$.

Visuellement, cela se traduit par la réalisation de trois cartes qui permettent une comparaison efficiente ${ }^{6}$. La première illustre la part de la population vivant dans un espace périurbain ou rural, autrement dit ici la part de la population vivant dans un espace sous gestion de la Gendarmerie nationale (cf. illustration 1). La seconde représente les catégories d'infractions constatées au sein des espaces urbains par département (les zones police). La troisième correspond aux catégories d'infractions constatées au sein des espaces ruraux et périurbains par département (zone gendarmerie).

Illustration 1 - Part d'individus vivant dans une « zone gendarmerie » par département en 2009

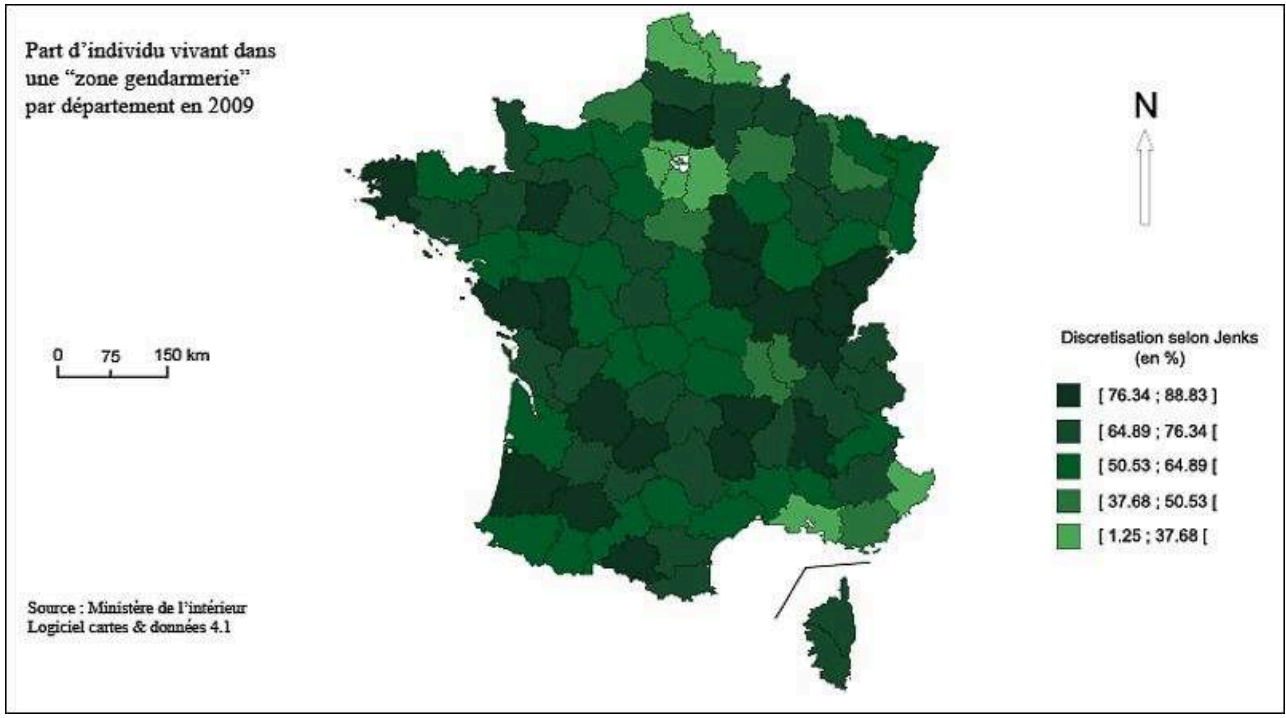

Source : Ministère de l'intérieur. 


\section{Quelle place de l'urbanité dans l'étude de la délinquance?}

\section{La répartition spatiale des catégories de délinquance}

La cartographie des catégories de délinquance permet d'établir une première distinction entre espace rural et périurbain d'une part et espace urbain d'autre part. Qualitativement, l'observation des deux cartes (avec la même légende) permet de déterminer les traits urbains ou non d'une catégorie de délinquance. En effet, certaines catégories présentent un taux par habitant particulièrement important dans les espaces urbains alors qu'ils sont moins prononcés dans les «zones gendarmerie", permettant la caractérisation d'une délinquance à primauté urbaine :

- la délinquance totale

- les crimes et délits contre les personnes

- les atteintes volontaires à l'intégrité physique (illustration 2)7

Illustration 2 - Cartes des taux d'atteintes volontaires à l'intégrité physique

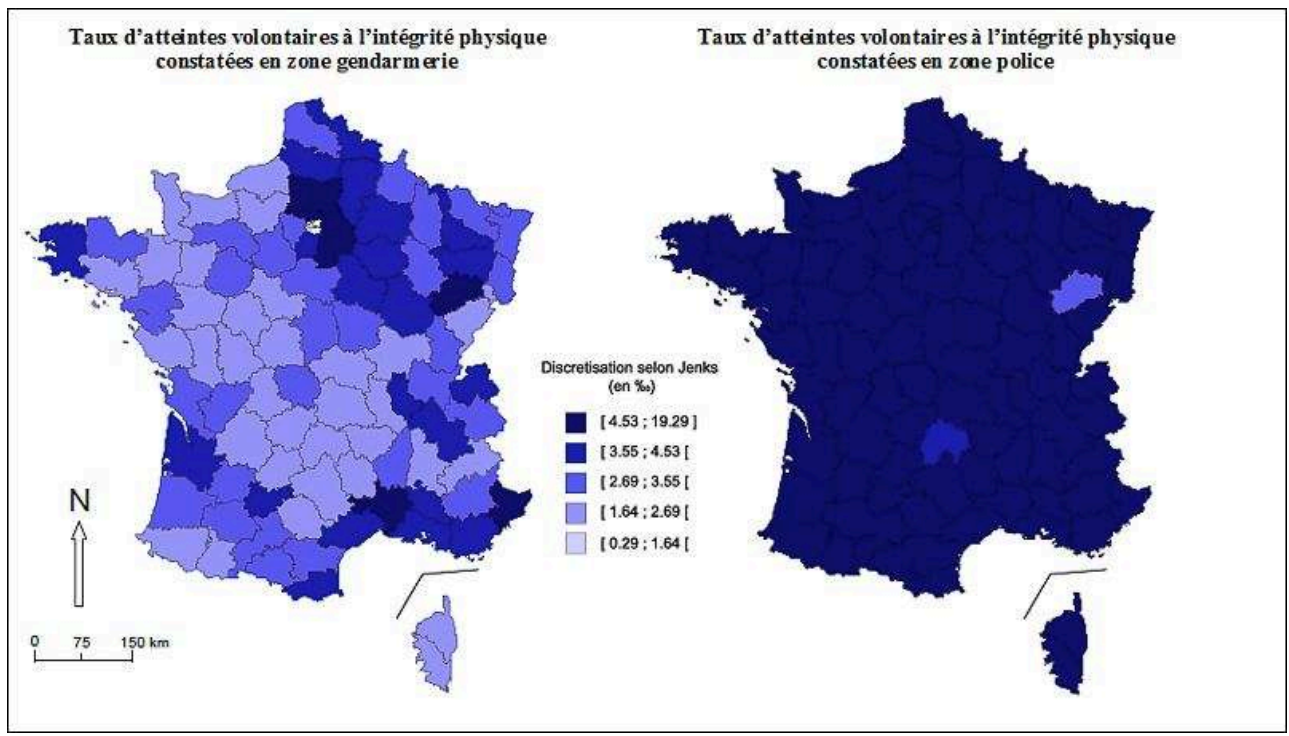

Auteur : J. Perrin, 2013.

Ces données ne signifient pas que ces catégories de délinquance soient absentes des campagnes, mais bien qu'elles sont plus récurrentes au sein des villes. Ce groupe d'une délinquance à primauté urbaine a été défini par la concentration des catégories au sein desquels $75 \%$ à $100 \%$ des occurrences se situent dans les deux classes les plus hautes et dont $50 \%$ se situent dans la classe la plus haute. Ces éléments permettent ainsi d'établir qu'en 2009 ces catégories de délinquance sont davantage urbaines et de manière généralisée pour les villes françaises, de Lille à Marseille et de Brest à Strasbourg.

Ensuite, deux autres catégories de délinquance constituent un ensemble de déviances négatives où l'influence de la ville semble très présente, mais de manière moins forte que dans la délinquance à primauté urbaine. Cette catégorie qualifiée de délinquance à dominante urbaine est formée par :

- les atteintes aux biens

- la délinquance de voie publique 
- les infractions révélées par l'action des services

- les vols (illustration 3)

Illustration 3 - Cartes des taux de vols

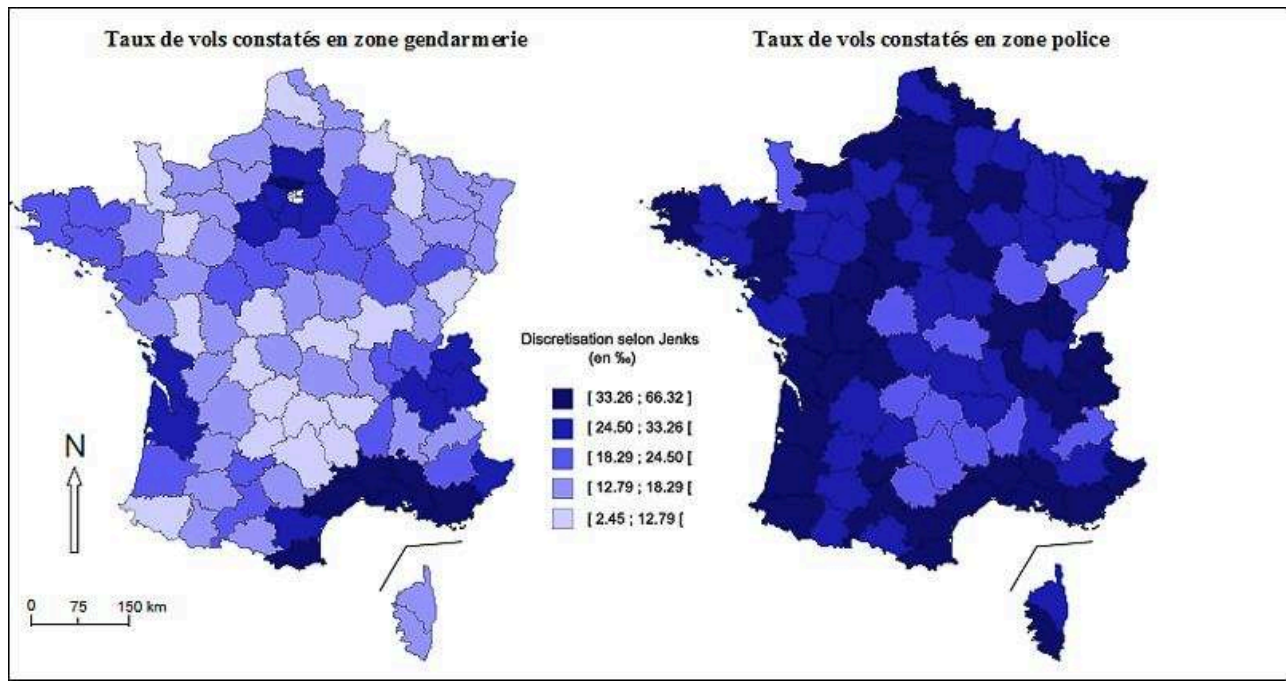

Auteur : J. Perrin, 2013.

Ce groupe rassemble les catégories au sein desquels $50 \%$ à $75 \%$ des occurrences se situent dans les deux classes les plus hautes et dont $50 \%$ se situent dans la classe la plus haute. Cette répartition n'est pas homogène sur l'ensemble des départements mais elle apparait tout de même présente dans certains espaces urbains allant de Pau à Chartres, de Rouen à Grenoble ou encore sur le pourtour méditerranéen de Nice à Perpignan.

A contrario, cette classification présente également une catégorie de délinquance marquée par une influence marginale de l'urbain, présentant ainsi une répartition plus mitigée entre les zones gendarmerie et police à savoir les escroqueries et infractions économiques et financières (illustration 4).

Illustration 4 - Cartes des taux d'escroqueries et d'infractions économiques et financières

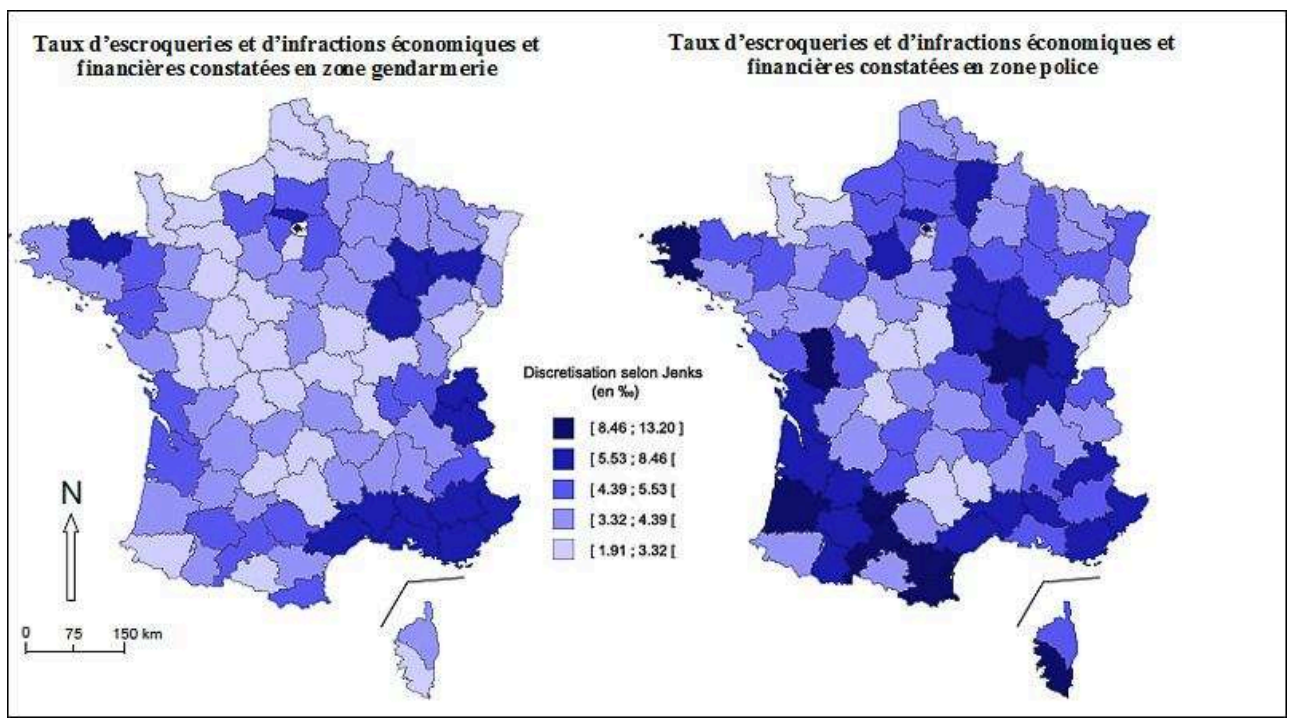

Auteur : J. Perrin, 2013. 

spatiale plus fine afin d'en cerner la complexité géographique. À nouveau, la lecture qualitative des cartes permet de déterminer l'influence de l'urbain dans les différentes infractions qui composent chaque catégorie de délinquance. Le tableau ci-dessous résume, à l'aide de trois exemples, cette décomposition des catégories de délinquance.

Illustration 5 - Influence de l'urbain dans différentes infractions

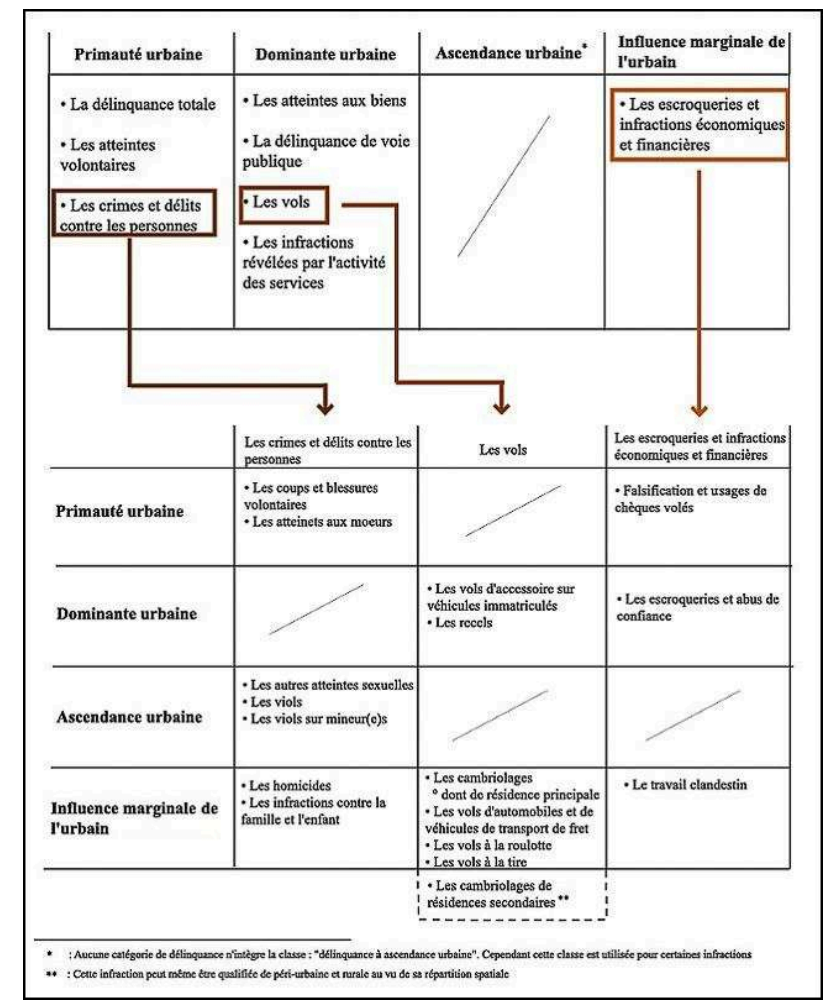

Auteur : J. Perrin, 2013.

Au sein des catégories de délinquance à primauté urbaine, la catégorie des crimes et délits contre les personnes laisse apparaître des infractions avec de fortes disparités spatiales. En effet, deux infractions sont marquées par une primauté urbaine :

- les atteintes aux mœurs

- les coups et blessures volontaires (illustrations 6) 
Illustration 6 - Cartes des taux de coups et blessures volontaires

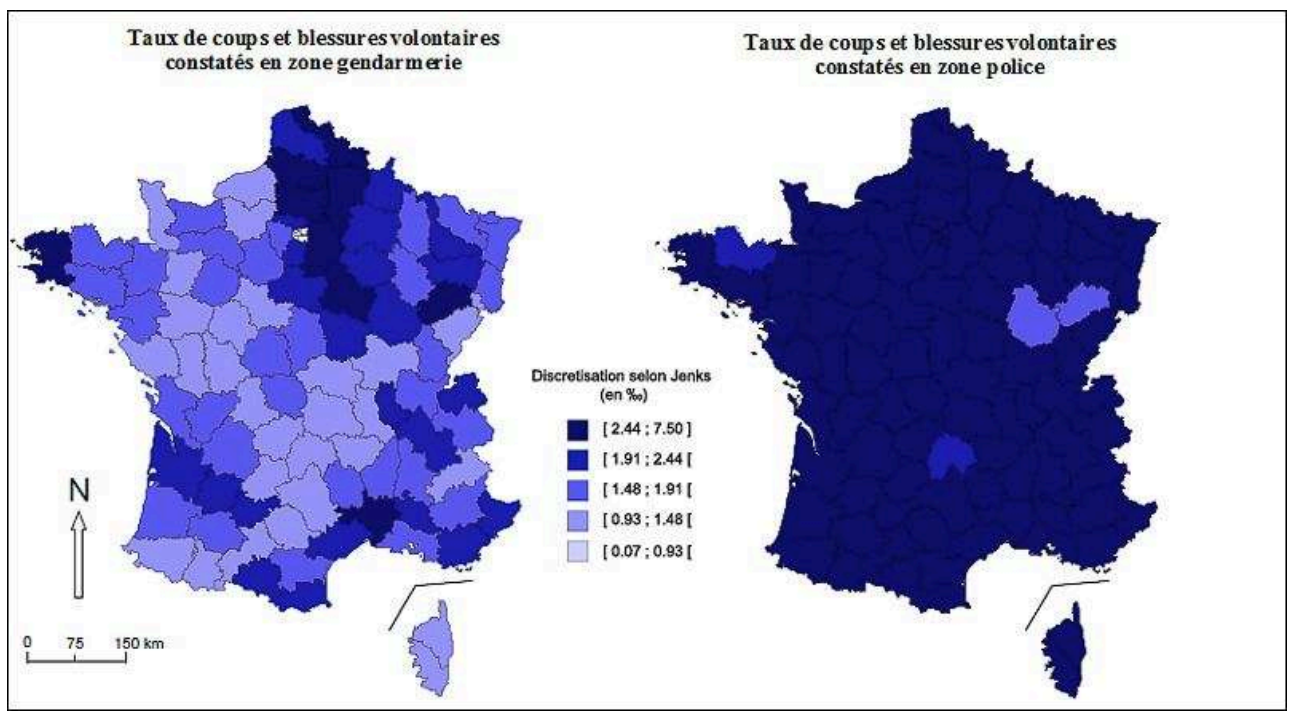

Auteur: J. Perrin, 2013.

33 D'autres infractions se caractérisent également par une influence de l'urbain, mais de manière plus diffuse que pour la délinquance à primauté ou à dominante urbaine. Il s'agit de la délinquance à ascendance urbaine :

- les autres atteintes sexuelles

- les viols sur mineur(e)s

- les viols (illustration 7)

Illustration 7 - Cartes des taux de viols

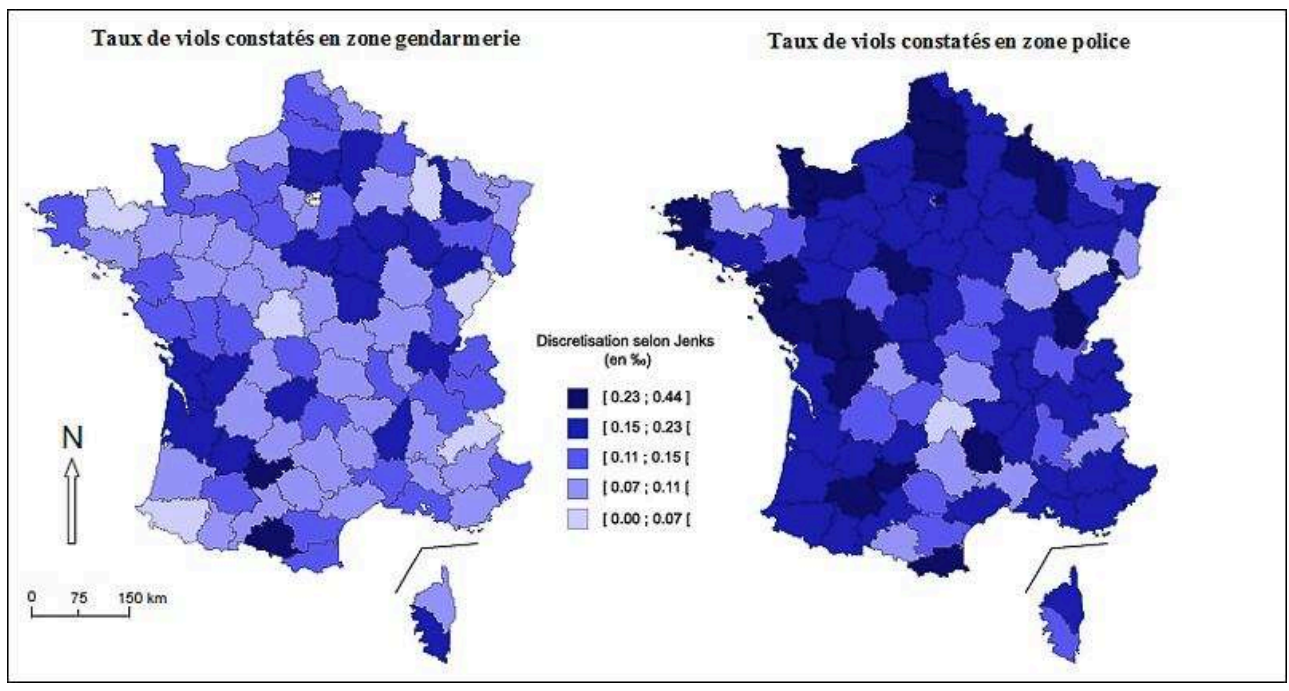

Auteur : J. Perrin, 2013.

34 Ce groupe d'une délinquance à ascendance urbaine concentre les catégories au sein desquels $25 \%$ à $50 \%$ des occurrences se situent dans les deux classes les plus hautes et dont $50 \%$ se situent dans la classe la plus haute. Cela s'observe spatialement avec des départements où les espaces urbains se situent dans la classe la plus haute (Mende, 
Lons-le-Saunier, etc.), complétés par un nombre important de villes de la seconde classe (Alençon, Orléans, Troyes, Avignon, Rochefort, etc.).

Enfin certaines infractions ne se caractérisent pas par une emprise spécifiquement urbaine et constituent ainsi une classe d'influence marginale de l'urbain :

- les infractions contre la famille et l'enfant

- les homicides (illustration 8)

Illustration 8 - Cartes des taux d'homicides

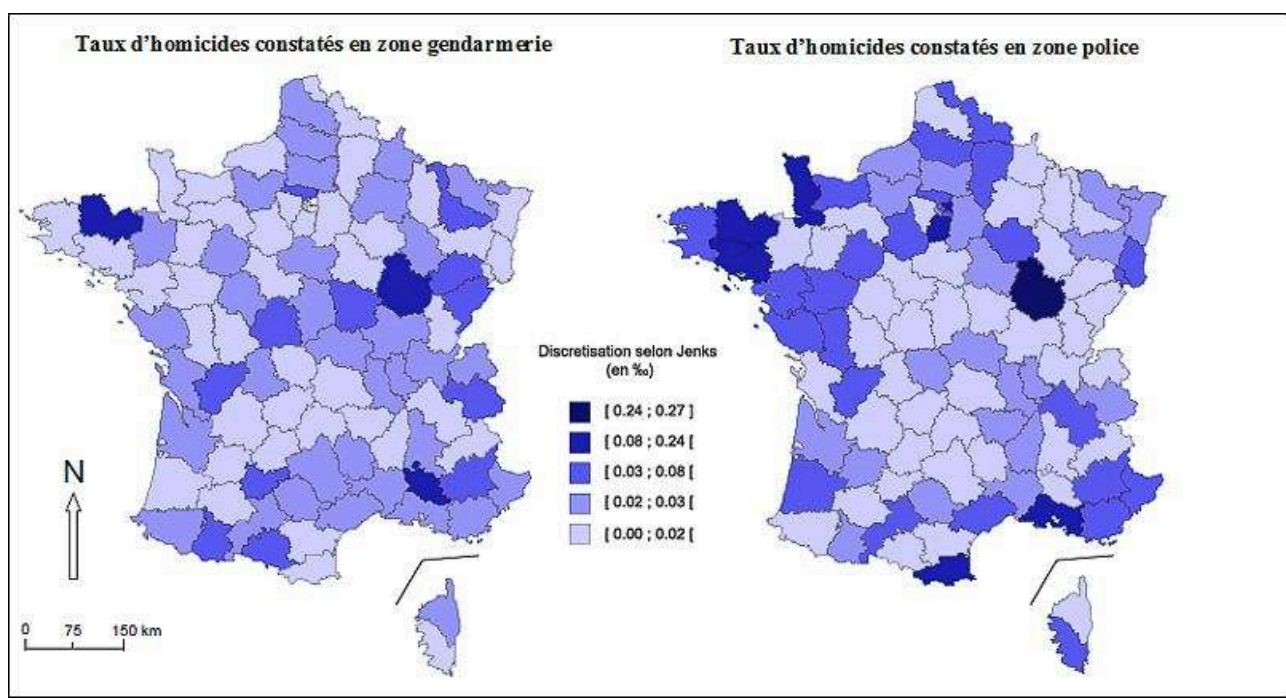

Auteur : J. Perrin, 2013.

Cet approfondissement de la catégorie des crimes et délits contre les personnes montre ainsi des infractions où l'emprise de l'urbain est particulièrement variée. Cependant, le poids que représente l'infraction «coups et blessures volontaires" dans le calcul de cette catégorie caractérise cette dernière comme la composante principale en délinquance à primauté urbaine. Cette analyse est reproductible avec plusieurs catégories de délinquance dont deux exemples sont présentés dans l'illustration cidessus.

Cette analyse permet ainsi d'établir une hiérarchie des crimes et délits en fonction de l'influence de la ville. Cependant, cette analyse se limite à une observation qualitative où les espaces ruraux, périurbains, ainsi que les espaces urbains, présentent des caractéristiques particulièrement variées. L'étude de la délinquance rurale sous influence urbaine nécessite une approche plus fine afin d'en cerner la complexité au travers d'une analyse de la délinquance au sein des espaces ruraux et périurbains.

\section{La délinquance périurbaine et rurale sous influence urbaine}

Les cartes de la délinquance constatée en zone gendarmerie permettent d'extraire des informations sur la localisation préférentielle de certains types de délinquance. De plus, la mise en relation de ces cartes avec celles montrant la part de la population vivant en milieu rural et périurbain permet d'établir des liens entre les espaces où le poids des villes est le plus grand et certains types de délinquance. L'analyse permet ainsi de 
distinguer plusieurs espaces ruraux et périurbains qui concentrent des délinquances spécifiques.

Tout d'abord, les infractions liées aux atteintes aux biens s'observent de manière préférentielle dans les départements suivants ${ }^{9}$ :

- les départements de l'Ile de France

- les départements du pourtour méditerranéen

- les départements de la région Rhône-Alpes et notamment le département du Rhône

- le département de la Gironde

Illustration 9 - Carte des taux d'atteintes aux biens constatées en zone gendarmerie

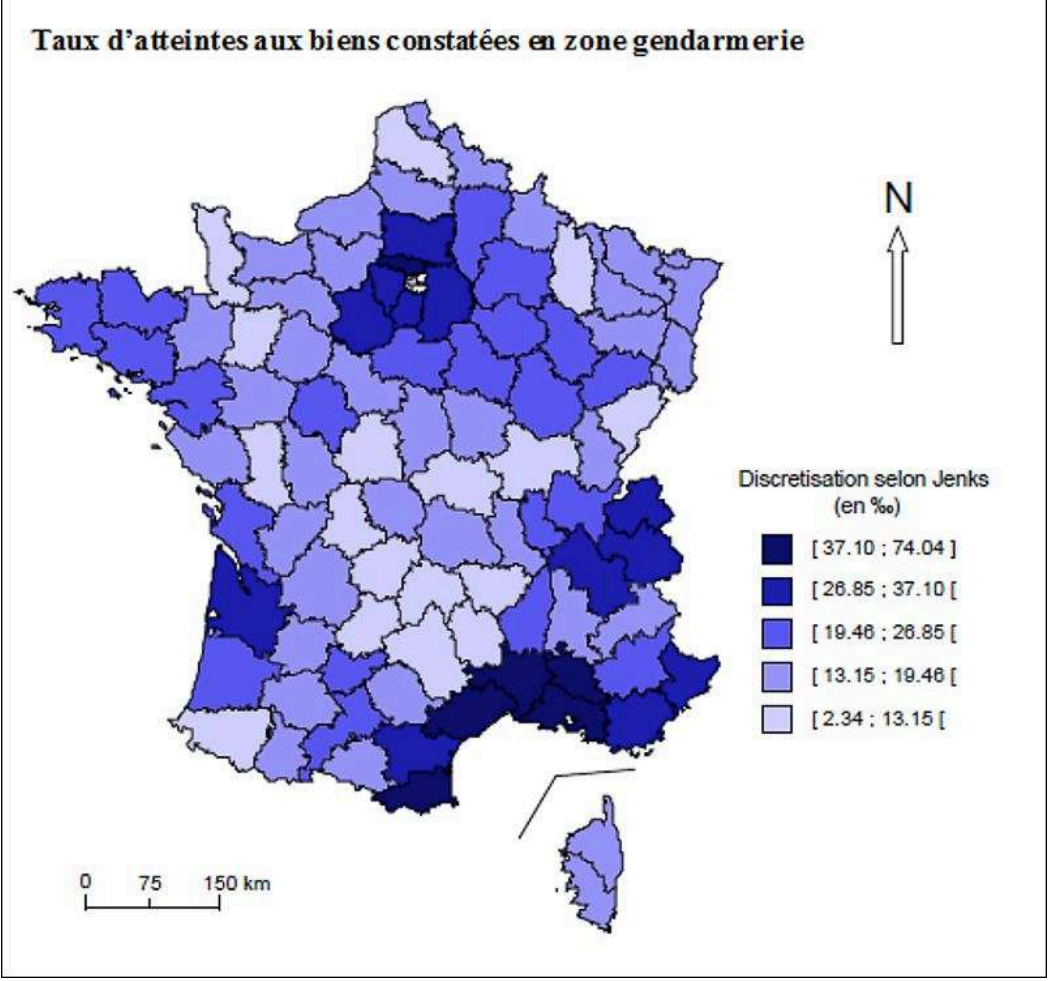

Auteur : J. Perrin, 2013.

Ce constat met clairement en avant l'importance de la ville, et notamment des plus grandes, dans les infractions des atteintes aux biens. En effet, ces départements sont marqués par une forte présence de la ville puisque les parts de la population vivant en milieu rural ou périurbain sont parmi les plus faibles (entre $1 \%$ et $27 \%$ ). Dans ces départements, les plus grandes villes tendent ainsi à diffuser « leur délinquance » dans une aire plus vaste au sein des territoires ruraux et périurbains qui composent le département. C'est le cas pour Bordeaux, Marseille, Montpellier, Pontoise, etc. Il est tout de même nécessaire de noter l'absence du Nord et de sa métropole Lille dans cette catégorie, malgré la cinquième place de Lille dans la hiérarchie des villes françaises pour son aire urbaine. Cette localisation préférentielle des infractions liées aux atteintes aux biens dans l'Ile de France et le sud-est se retrouve dans les enquêtes de victimation qui montrent également des valeurs supérieures à la moyenne (Rizk, 2009). Toutefois, ces espaces se caractérisent par une forte attractivité touristique qui draine également de nombreux délinquants attirés par le potentiel de vols pendant quelques semaines de l'année, faisant de fait augmenter les chiffres. 
41 Ensuite, les catégories de délinquance qui englobent les atteintes aux personnes se concentrent quant à elles dans des espaces également spécifiques ${ }^{10}$ :

- les départements de l'Ile de France

- les départements du pourtour méditerranéen

- le département du Rhône

- le département du Finistère

- les départements situés dans un axe nord-sud allant de l'Yonne voire de la Côte d'Or jusque dans le département du Nord

Illustration 10 - Carte des taux de coups et blessures volontaires en zone gendarmerie

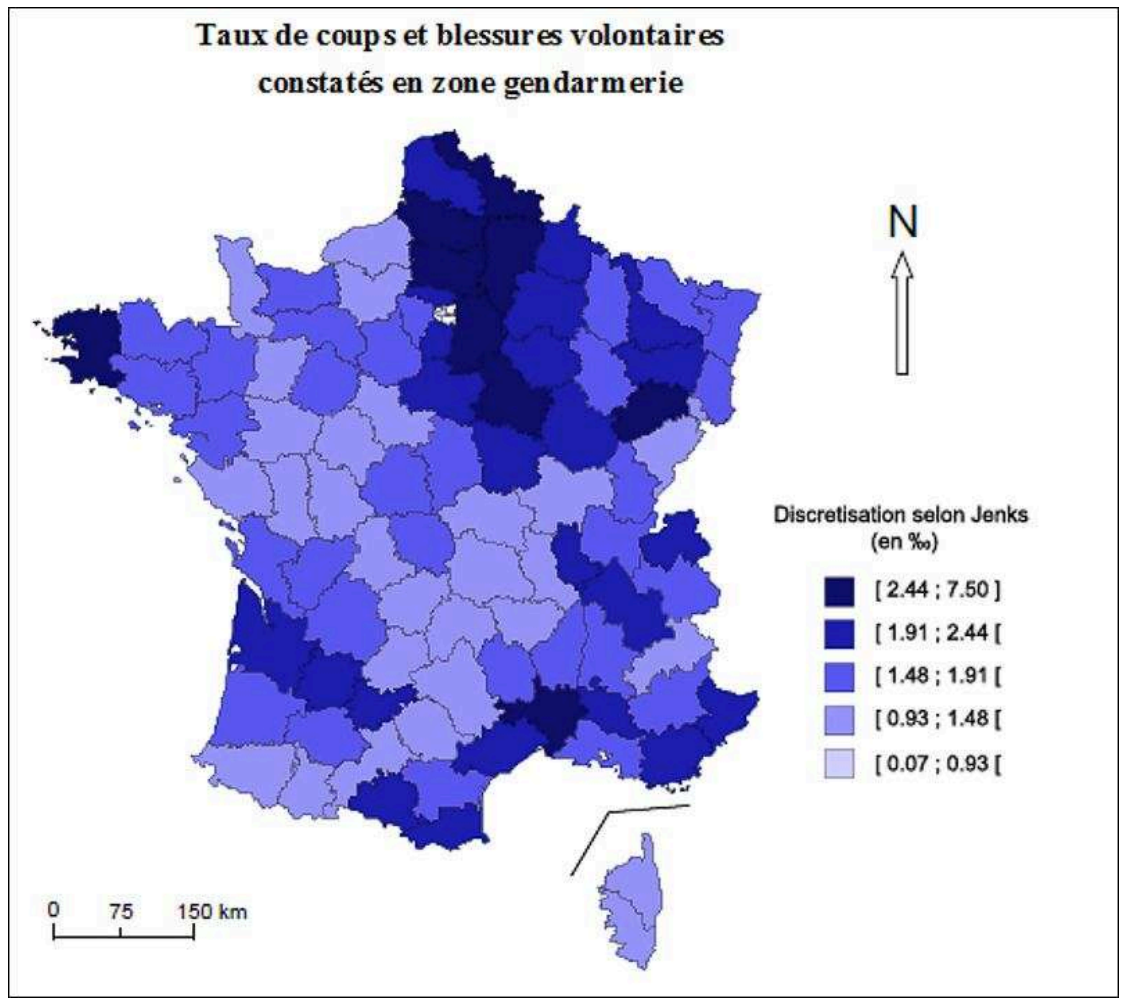

Auteur : J. Perrin, 2013.

À nouveau, les départements les plus urbanisés se confondent avec ceux où la délinquance contre les personnes est la plus importante. Mais certains départements apparaissent alors qu'ils ne se caractérisent pas par une forte urbanisation. C'est le cas duFinistère, de l'Yonne ou encore de l'Oise ; cependant, ces deux derniers subissent l'influence de Paris et de sa région (Guiberteau et al., 2009) (Lecrenais, 2013).

Enfin, les types de délinquance en lien avec les stupéfiants suivent des logiques de localisations particulières où une lecture supranationale se révèle nécessaire. En effet, les divers trafics ou reventes de produits stupéfiants s'observent en relation avec le trafic mondial et européen de la drogue. Ainsi, les départements les plus touchés sont:

- les départements de la région Lorraine

- les départements frontaliers avec l'Espagne, l'Italie et la Belgique 
Illustration 11 - Carte des taux de trafics ou reventes de stupéfiants sans usage constatés en zone gendarmerie

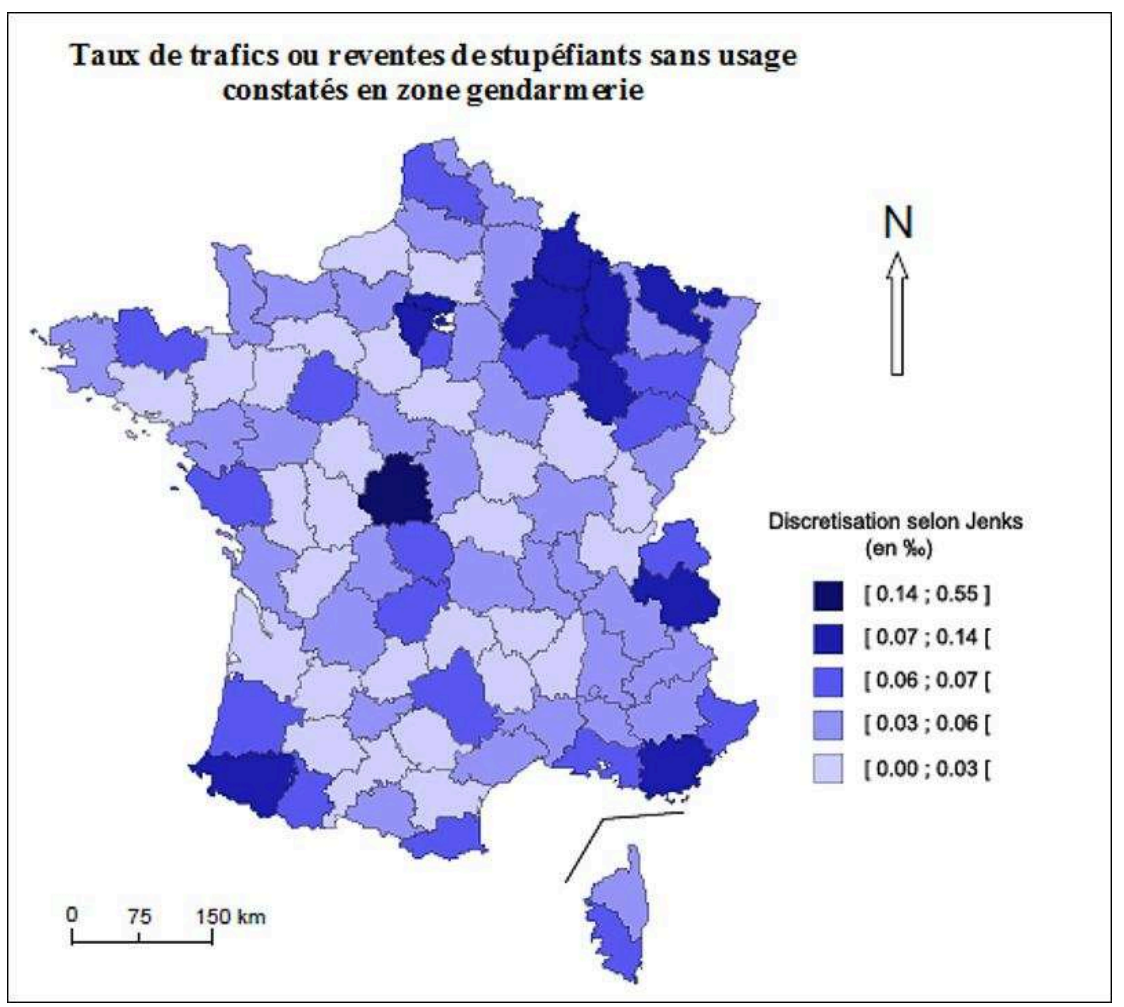

Auteur : J. Perrin, 2013. utilisés par les trafiquants de drogues et notamment les go fast qui correspondent à des véhicules très rapides et largement chargés de drogue qui remontent du sud de l'Espagne et de l'Italie jusqu'en France. D'autre part, le Nord-Est de la France est quant à lui affecté par la proximité avec les Pays-Bas qui pratiquent une législation plus laxiste sur les drogues dites douces comme le cannabis, créant ainsi de véritables réseaux parallèles qui traversent les frontières.

\section{Conclusion}

Le paradoxe du décalage entre l'imaginaire territorialisé de la délinquance et la quasiabsence de la géographie parmi les sciences traitant de la délinquance est particulièrement prégnant en France. La géographie n'en reste pas moins une discipline disposant d'un large potentiel pouvant participer au développement des connaissances de cette thématique. Malgré les limites de la cartographie statistique, celle-ci reste un outil indispensable pour le géographe afin de prendre part aux travaux portant sur les déviances négatives. Cette porte d'entrée graphique ouvre de nombreuses possibilités d'étude qui sont à mener afin de découvrir puis d'approfondir les caractéristiques spatiales de la délinquance.

La répartition spatiale des déviances négatives apparaît ainsi affectée par la mondialisation du territoire français. D'une part, la concentration de certaines infractions dans les espaces soumis à la compétition urbaine et au creusement des inégalités sociales et spatiales laisse transparaître une métropolisation de la 
délinquance. D'autre part, d'autres infractions, comme celles liées aux produits stupéfiants, impliquent une lecture transnationale de la délinquance. Il devient nécessaire d'intégrer l'effet des frontières, ainsi que les différentiels législatifs qui y sont associés, mais aussi les logiques réticulaires efficientes à l'échelle mondiale. Cette étude qui établit des tendances de localisation préférentielle de certaines catégories de délinquance et d'infractions à l'échelle des départements français en 2009 ouvre de nouveaux questionnements. En effet, il apparaît nécessaire de mettre en place une réflexion sur les échelles pertinentes afin de cerner les recompositions de la délinquance. L'échelle départementale nous permet d'établir des tendances générales sur une année. Cependant, sans complément, elle reste insuffisante pour cerner les influences locales qui nous permettraient de nous rapprocher davantage de la réalité géographique.

D'autres questionnements émergent en lien avec cette "nouvelle délinquance » au travers des effets de la mondialisation, de l'ouverture des frontières, des choix politiques, ou encore des orientations mises en place par les autorités administratives et par les professionnels. Par ailleurs, les coûts de la mobilité, le choix ou la contrainte résidentielle, ainsi que l'accessibilité apparaissent comme autant de pistes à explorer pour les géographes, permettant de faire la jonction entre l'étude de la ségrégation dans les territoires périmétropolitains et l'étude de la délinquance.

\section{BIBLIOGRAPHIE}

Bauer A., 2006. Géographie de la France criminelle. Paris, Odile Jacob, 278 p.

Besson J.-L., 2004. Les cartes du crime. Paris, Presses Universitaires de France, 288 p.

Bui Trong L., 1998. Sur quelques secrets de fabrication. Les cahiers de la sécurité intérieure, $\mathrm{n}^{\circ} 33$, p. 225-233.

Brunet R, 1989. Redéploiements de la géographie. Espaces Temps, vol. 40-41, p. 61-68.

Camilleri G., Lazerges C., 1992. Atlas de la criminalité en France. Montpellier, Paris, Reclus/La documentation française, $160 \mathrm{p}$.

Cauvin C., Escobar F., Serradj A., 2008. Cartographie thématique 3 : méthodes quantitatives et transformations attributaires. Paris, Hermès science/Lavoisier, $450 \mathrm{p}$.

Champagne P., 1993. La vision médiatique. In Bourdieu P., La misère du monde, Paris, Édition du Seuil, p. 61-79.

Dieu F., 2002. La production de sécurité dans les territoires ruraux français. Les cahiers de la sécurité intérieure, n 50, p. 149-167.

Dieu F, 2009. Aménagement du territoire et sécurité. Les cahiers de la sécurité, n 8, p. 43-48.

Frémont A., 2005. Aimez-vous la géographie ? Paris, Flammarion, p. 42-43.

Garcin P., 2005. Sécurité, insécurité. Bilan, attentes, clés pour une stratégie globale. Paris, Armand Colin, $313 \mathrm{p}$. 
Ghorra-Gobin C. (dir.), 2011. Géographie et éthique. Géographie et Cultures, n 7475, 280 p.

Gregory D. (dir.), 2009. The dictionary of human geography. Cinquième édition. Hoboken, WileyBlackwell, 120 p.

Guiberteau V., Le Scouezec P., Mierlot J.-M., et al., 2009. Déplacement domicile-travail dans l'Oise. Analyse, $\mathrm{n}^{\circ}$ 35, Insee, Dréal, $6 \mathrm{p}$.

Kelling G.L., Coles C., 1996. Fixing Broken Windows: Restoring Order and Reducing Crime in Our Communities. Detroit, Free Press, 336 p.

Lacoste Y, 2003. Article délinquance. De la géopolitique aux paysages. Dictionnaire de la géographie. Paris, Armand Colin, p. 115.

Lecrenais C., 2013. Dans l'Yonne, les actes de la vie quotidienne dessinent 17 territoires. Bourgogne dimensions, $\mathrm{n}^{\circ} 184$, Insee, $4 \mathrm{p}$.

Mattelly J.-H., 2007. Police : des chiffres et des doutes. Paris, Editions Michalons, 276 p.

Mayhew S., 2009. A dictionary of geography. Quatrième édition. Oxford, Oxford University Press, $111 \mathrm{p}$.

Moisi D., 2008. La géopolitique de l'émotion. Paris, Flammarion, 267 p.

Monmonier M., 1991. Comment faire mentir les cartes, ou du mauvais usage de la géographie. Paris, Flammarion, $232 \mathrm{p}$.

Mouhanna C., 2001. Faire le gendarme : de la souplesse informelle à la rigueur bureaucratique. Revue française de sociologie, vol. 42, numéro 42-1, p. 31-55.

Mucchielli L, 2002. Violences et insécurité. Fantasmes et réalité dans le débat français. Paris, La Découverte, $158 \mathrm{p}$.

Noseda V., 2005. Violences urbaines. Une exploration au-delà des interprétations reçues. Travaux et recherches, $\mathrm{n}^{\circ}$ 29. Lausanne, Institut de géographie, $142 \mathrm{p}$.

Perrin J., 2013. La délinquance rurale sous influence urbaine. La nouvelle délinquance observatoire des recompositions des relations villes-campagnes. Strasbourg, Université de Strasbourg, Mémoire de recherche en géographie, $255 \mathrm{p}$.

Pigeon P., 2005. Géographie critique des risques. Paris, Economica, 217 p.

Rebotier J., 2008. Les territorialités du risque urbain à Caracas. Les implications d'un construit sociospatial dans une métropole d'Amérique latine. Thèse de géographie, Paris, Université Paris III, 429 p.

Rémy B., 2000. La délinquance à la Réunion. Approche géographique d'une situation dans un espace insulaire. Paris, L'Harmattan, $272 \mathrm{p}$.

Rizk C., 2009. Victimisation et délinquance enregistrées dans huit grands ensembles régionaux. Géographie comparée des vols liés aux véhicules et des vols dans les résidences des ménages. Les cahiers de la sécurité, $\mathrm{n}^{\circ} 8, \mathrm{p} .52-62$.

Robert P. et al., 1994. Les comptes du crime. Paris, L'Harmattan, 329 p.

Robert P., 2003. L'insécurité en France. Paris, La Découverte, 119 p.

Roché S., 2002. Tolérance zéro. Incivilités et insécurité. Paris, Odile Jacob, 303 p.

Winick C., 1978. Deviance and the mass media. Londres, Sage, 305 p. 


\section{NOTES}

1. Si la géographie anglo-saxonne présente des entrées sur les thématiques délictuelles dans divers dictionnaires (Mayhew, 2009), (Gregory (dir.), 2009), il apparait ici nécessaire de souligner la quasi absence de ce type d'occurrence dans les dictionnaires français.

2. À noter également l'ouvrage Géographie de la France criminelle d'Alain Bauer (Bauer, 2006), ancien président $\mathrm{du}$ conseil d'orientation de l'observatoire national de la délinquance et des réponses pénales. Cet ouvrage, au vu de sa lisibilité et sa scientificité, ne se présente pas comme un ouvrage de référence de ce questionnement géographique.

3. C'est pourquoi, la géographe se doit d'expliciter ses choix successifs afin de démontrer la partialité de la carte et le respect des règles (du moins de ses structures rigides).

4. La Gendarmerie nationale travaille sur ces questionnements afin de s'adapter aux mieux à ces transformations comme en témoigne le colloque «Vieillissement et territoires : quels enjeux pour la gendarmerie ?» organisé le 8 février 2011 par le Centre de recherche de la Gendarmerie nationale.

5. Le choix d'utiliser cette partition entre zone rurale et périurbaine d'une part et urbaine d'autre part (plutôt que celle établie par l'INSEE) permet de conserver la concordance spatiale des données statistiques et démographiques. En effet, la répartition des compétences entre les espaces sous contrôle de la police ou de la gendarmerie ne suit pas nécessairement le découpage de l'INSEE. Ainsi, l'étude se base sur les publications du ministère de l'Intérieur qui apportent les éléments de statistiques délictuelles et démographiques pour un même espace (Aspects de la criminalité et de la délinquance constatées en France, 2009, La documentation française).

6. Le discrétisation utilisée est fondée sur la notion de variance. En effet, dite de "Jenks ", elle permet par une procédure itérative de maximiser l'homogénéité des classes tout en minimisant la variance entre les classes. Les limites des classes sont formées par les valeurs observées de la variable pouvant ainsi illustrer d'éventuelles ruptures. Au final, cette méthode permet de minimiser l'erreur due à la généralisation tout en maximisant l'homogénéité de classes (Cauvin et al., 2008).

7. Pour des raisons de lecture et de clarté nous ne présenterons qu'un exemple de catégorie de délinquance par classe (ici les atteintes volontaires à l'intégrité physique)

8. Cette analyse reprend les éléments géographiques les plus pertinents. Une analyse plus développée a été réalisée (Perrin, 2013).

9. Les infractions qui suivent cette répartition spatiale sont : la délinquance de voie publique, les vols, les vols d'accessoires sur véhicules immatriculés, les cambriolages, les cambriolages de résidences principales, les vols d'automobiles et de véhicules de transports de fret, les vols à la roulotte, les vols à la tire, et les destructions et dégradations.

10. Les infractions concernées sont les atteintes volontaires à l'intégrité physique, les crimes et délits contre les personnes, les coups et blessures volontaires, les autres atteintes sexuelles, les infractions contre la famille et l'enfant. Pour des raisons de lisibilité et de clarté, nous présentons un exemple représentatif de ces infractions (les coups et blessures volontaires).

\section{RÉSUMÉS}

Les caractéristiques spatiales de la délinquance, bien que présentes dans le discours politicomédiatique, restent très marginalement étudiées par la géographie française, contrairement à la 
géographie anglo-saxonne. Or, les transformations à l'œuvre dans les territoires français, sous l'action de la métropolisation, affectent ces caractéristiques spatiales et les représentations de la délinquance qui y sont associées. La cartographie statistique et les limites qui lui sont propres participent aux diverses possibilités de lecture, d'analyse et de communication des phénomènes délictuels dans une perspective géographique. A rebours des multiples instrumentalisations de l'insécurité, la délinquance apparaît dans une perspective géographique comme un observatoire permettant d'analyser l'influence de l'urbain sur les territoires ruraux et périurbains. Cet article, sans pouvoir prétendre à l'exhaustivité d'une thématique aussi vaste, présente des pistes de lecture et d'analyse de ce processus en s'appuyant sur une analyse statistique et une cartographie comparées de la délinquance dans les zones police et dans les zones gendarmerie.

Delinquency's spatial characteristics, although present in the politico-media speech, remain marginally studied by the French geographers, on the opposite of the Anglo-Saxon geography. However, the transformations at work in the French territories, under the action of the metropolization, affect these spatial characteristics of the delinquency as well as its representations. The statistical mapping and the limits of its application, give way to various interpretations, analyses and communication of the criminal phenomena from the geographical point of view. Contrary to the multiple instrumentalizations of insecurity, delinquency appears, from the geographical point of view, like an observatory making it possible to analyze urban influence on rural and suburban territories. This paper offers some elements to read and analyze the various aspects of this phenomenon, based on a compared statistical analysis and cartography of the delinquency in the police and gendarmerie areas.

\section{INDEX}

Keywords : delinquency, criminality, urban-rural connections, crime mapping, urban influence Mots-clés : délinquance, criminalité, relations villes-campagnes, cartographie criminelle, influence urbaine

\section{AUTEUR}

\section{JORICK PERRIN}

Jorick Perrin, jorick.perrin@etu.unistra.fr, est étudiant en master enseignement d'histoiregéographie à l'Université de Strasbourg. Ses travaux sont dirigés par Géraldine Djament-Tran et il a publié récemment :

- Perrin J., 2013. La délinquance rurale sous influence urbaine. La nouvelle délinquance observatoire des recompositions des relations villes-campagnes. Strasbourg, Université de Strasbourg, mémoire de recherche en géographie, $255 \mathrm{p}$. 